Applied Mathematical Sciences, Vol. 8, 2014, no. 106, 5293 - 5302

HIKARI Ltd, www.m-hikari.com

http://dx.doi.org/10.12988/ams.2014.47528

\title{
A Boussinesq-type Model for Waves Generated by Flow over a Bump
}

\author{
L.H. Wiryanto \\ Department of Mathematics, Bandung Institute of Technology \\ Jalan Ganesha 10, Bandung 40132, Indonesia \\ Sudi Mungkasi \\ Department of Mathematics, Sanata Dharma University \\ Mrican, Tromol Pos 29, Yogyakarta 55002, Indonesia
}

Copyright (C) 2014 L. H. Wiryanto and Sudi Mungkasi. This is an open access article distributed under the Creative Commons Attribution License, which permits unrestricted use, distribution, and reproduction in any medium, provided the original work is properly cited.

\begin{abstract}
A uniform flow disturbed by a bump is studied. The effect of the disturbance is presented at the surface, generating wave. The wave propagation is modeled into a couple of equations, in terms of the surface elevation and the depth average velocity. The numerical solution of the equations is simulated to observe the propagation, especially for long run time, using a predictor-corrector method. A steady solitary surface profile is obtained for supercritical upstream flow, similarly for subcritical flow but for negative amplitude. In the transient process, more waves are generated but some of them propagates to the left or right, and only one wave remains above the bump.
\end{abstract}

Mathematics Subject Classification: 35C20, 76B07, 76B25

Keywords: Boussinesq equations, fKdV equation, predictor-corrector method

\section{Introduction}

A 2-D flow is considered over a bump on the bottom of a channel. The fluid is assumed to be ideal and the flow is irrotational, so that the flow can be 
described in terms of potential function. Far upstream the flow is uniform with velocity $U_{0}$ and depth $H$. The effect of the bump is to generate waves propagating upstream or downstream from the bump.

We examine the unsteady problem, formulated as Boussinesq-type equations, and solve numerically. Our results indicate that the steady wave is set up when the upstream flow is supercritical, i.e. the Froude number $F=U_{0} / \sqrt{g H}$ greater than 1. Here $g$ is the acceleration of gravity. The solution is like a solitary wave. This steady wave agrees with one of the solutions obtained by some researchers, such as Forbes and Schwartz [1], Vanden-Broeck [2] and Forbes [3], who solved numerically the steady problem, direct from the exact equations, without approximating the governing equations into a set of equations. Their numerical computations indicated that there were two branches of solutions for $F$ greater than a certain number and greater than 1 .

Another approach, the steady problem is approximated into a forced Korteweg de Vries (fKdV) equation. The model contains one non-dimensional parameter Froude number $F$, and the forcing term representing the bump. Shen et al. [4] derived the fKdV equation, based on Euler equations. Similarly, for two fluid system, Shen [5] derived the model having a fKdV-type equation, both for interfacial wave and the surface wave. Shen et al. [4] confirm their results to Forbes and Schwartz [1], two solutions of steady solitary wave. In case the forcing term is a secant-hyperbolic bump, corresponding to the solution of the KdV, Chardar et al. [6] as well as Camassa and Wu [7, 8] obtained one solution of secant-hyperbolic-type, only for a certain Froude number depending on the height and width of the bump.

The difference between these two and one solution is then observed by Wiryanto [9]. They derived the model in a form of the fKdV equation, based on the perturbation of the potential function from the uniform flow, and observes the effect of the bump width. The two steady solitary waves tend to a unique solution for a certain number of the width. Less than that number, two solutions with different amplitude are obtained.

Which solution is stable is our concern in this paper, together with our observation for subcritical flow. The problem is modeled for unsteady flow into Boussinesq-type equations, instead of an unsteady fKdV equation. We derive the model based on a small parameter related to the wave amplitude. The equations are then solved numerically by a predictor-corrector method. Wiryanto [10] also used a similar method to solve a model for interfacial wave. The simulation of the wave generation is performed to observe the propagation, for various Froude numbers and bottom topography. For long run, some part of the wave travels away, and a steady profile is performed, which confirms to one of the solutions from the steady fKdV equation. 


\section{Boussinesq-type Model}

The configuration of the flow over a bump is illustrated in Figure 1. As the system of coordinates, we choose Cartesian with the horizontal $\bar{x}$-axis along the undisturbed free surface, and the vertical $\bar{y}$-axis perpendicular to the horizontal axis. The bump is described by $\bar{y}=-H+\bar{h}(\bar{x})$. The surface elevation is $\bar{y}=\bar{\eta}(\bar{x}, \bar{t})$, with uniform depth $H$, as $\bar{x} \rightarrow-\infty$, and the potential function is denoted by $\bar{\phi}$.

The problem is mainly to determine the potential function $\bar{\phi}$ satisfying

$$
\bar{\phi}_{\bar{x} \bar{x}}+\bar{\phi}_{\bar{y} \bar{y}}=0
$$

in the flow domain $-\infty<\bar{x}<\infty,-H+\bar{h}(\bar{x})<\bar{y}<\bar{\eta}(\bar{x}, \bar{t})$; with conditions

$$
\begin{gathered}
\bar{\phi}_{\bar{y}}-\bar{\eta}_{\bar{x}} \bar{\phi}_{\bar{x}}-\bar{\eta}_{\bar{t}}=0, \\
\bar{\phi}_{\bar{t}}+\frac{1}{2}\left(\bar{\phi}_{\bar{x}}^{2}+\bar{\phi}_{\bar{y}}^{2}\right)+g \bar{\eta}=\frac{1}{2} U_{0}^{2},
\end{gathered}
$$

along the surface $\bar{y}=\bar{\eta}(\bar{x}, \bar{t})$, and

$$
\bar{\phi}_{\bar{y}}-\bar{\phi}_{\bar{x}} \bar{h}_{\bar{x}}=0
$$

along the bottom $\bar{y}=-H+\bar{h}(\bar{x})$. The right hand side of (3) is the situation for the uniform flow far upstream.

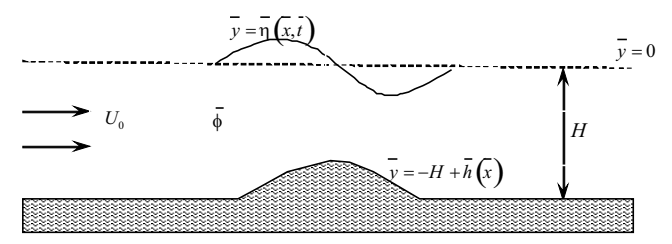

Figure 1: Sketch of the flow and coordinates.

Since the potential function tends to uniform flow, written as $\bar{\phi} \rightarrow U_{0} \bar{x}$, for $\bar{x} \rightarrow-\infty$, we express the potential function as a perturbation of the uniform flow, in terms of $\bar{\phi}=U_{0} \bar{x}+\bar{\Phi}(\bar{x}, \bar{y}, \bar{t})$. The governing equations (1)-(4) become respectively

$$
\bar{\Phi}_{\bar{x} \bar{x}}+\bar{\Phi}_{\bar{y} \bar{y}}=0
$$

subject to

$$
\begin{gathered}
\bar{\Phi}_{\bar{y}}-\bar{\eta}_{\bar{x}}\left(U_{0}+\bar{\Phi}_{\bar{x}}\right)=0 \\
\bar{\Phi}_{\bar{t}}+U_{0} \bar{\Phi}_{\bar{x}}+\frac{1}{2}\left(\bar{\Phi}_{\bar{x}}^{2}+\bar{\Phi}_{\bar{y}}^{2}\right)+g \bar{\eta}=0
\end{gathered}
$$


and

$$
\bar{\Phi}_{\bar{y}}-\bar{h}_{\bar{x}}\left(U_{0}+\bar{\Phi}_{\bar{x}}\right)=0
$$

The wave, which is generated by the disturbance of the flow, is expressed by the elevation of the surface as $\bar{y}=\bar{\eta}(\bar{x}, \bar{t})$, with assuming the amplitude $a$ and wavelength $\lambda$. These quantities are then used to scale the variables, together with other quantities, in obtaining the dominant order. To do so, we first define

$$
\bar{x}=\frac{x}{\lambda}, \quad \bar{y}=\frac{y}{H}, \quad \bar{t}=\frac{\sqrt{g H}}{\lambda} t, \bar{\eta}=\frac{\eta}{a}, \quad \bar{\Phi}=\frac{H}{\lambda a U_{0}} \Phi, \quad \bar{h}=\frac{h}{a} .
$$

Therefore, (5)-(8) become

$$
\mu^{2} \Phi_{x x}+\Phi_{y y}=0
$$

subject to

$$
\begin{gathered}
F \Phi_{y}-\mu^{2} F \eta_{x}\left(1+\epsilon \Phi_{x}\right)-\mu^{2} \eta_{x}=0, \\
F \Phi_{t}+F^{2} \Phi_{x}+\frac{1}{2} \epsilon F^{2}\left(\Phi_{\bar{x}}^{2}+\frac{1}{\mu^{2}} \Phi_{y}^{2}\right)+\eta=0,
\end{gathered}
$$

at the free boundary $y=\epsilon \eta$, and

$$
\Phi_{y}-\mu^{2} h_{x}\left(1+\epsilon \Phi_{x}\right)=0
$$

at the solid boundary $y=-1+\epsilon h(x)$. Here, two small parameters are defined as $\epsilon=a / H$ and $\mu=H / \lambda$.

The next step is to expand the potential function $\Phi$ in series of $\mu^{2}$

$$
\Phi=\Phi_{0}+\mu^{2} \Phi_{1}+\mu^{4} \Phi_{2}+\cdots,
$$

and substituted into (9). It is followed by using the solid boundary (12), so that we have

$$
\Phi=\Phi_{0}(x, t)+\mu^{2}\left[-\frac{1}{2} \Phi_{0_{x x}}(y+1-\epsilon h)^{2}+h_{x} y+\phi_{1}(x, t)\right]+O\left(\mu^{4}\right) .
$$

In this step we separate the variable $y$ from the unknown functions $\Phi_{0}$ and $\phi_{1}$, so that it can help us in applying to the free boundaries (10) and (11).

When (14) is substituted into (10) and also (11), the leading order of $\mu$ is respectively

$$
\begin{gathered}
F\left\{-\Phi_{0_{x x}}+\epsilon\left(-\Phi_{0_{x x}} \eta+\Phi_{0_{x x}} h\right)\right\}+F h_{x}-F \eta_{x}-\epsilon F \eta_{x} \Phi_{0_{x x x}}-\eta_{t}=0, \\
F \Phi_{0_{t}}+F^{2} \Phi_{0_{x}}+\frac{1}{2} F^{2} \epsilon \Phi_{0_{x}}^{2}+\eta=0 .
\end{gathered}
$$


In expressing (15) and (16) into physical variables, we define the depth average velocity

$$
u=\frac{1}{\epsilon \eta+1-\epsilon h} \int_{-1+\epsilon h}^{\epsilon \eta} \Phi_{x}(x, y, t) d y .
$$

The approximation of $u$ for the leading order of $\mu$ is $u \approx \Phi_{0_{x}}$, so that (15) and (16) become

$$
\begin{gathered}
\eta_{t}+F\left(\eta_{x}+u_{x}-h_{x}\right)+\epsilon F\left(\eta_{x} u_{x x}+u_{x} \eta-u_{x} h\right)=0 \\
u_{t}+F u_{x}+\frac{1}{F} \eta_{x}+\epsilon F u u_{x}=0 .
\end{gathered}
$$

We obtain (17) by differentiating (16) with respect to $x$, before replacing $\Phi_{0_{x}}$ by $u$. Equations (17) and (18) are the model of our problem.

In case $\epsilon=0$, the linear model can be solved for $t \rightarrow \infty$ as the steady solution. The surface elevation is proportional to the bottom topography. The steady linear equations of (17) and (18) can be solved analytically, by eliminating $u$ and then integrating with respect to $x$. Since the condition $\eta \rightarrow 0$ as $|x| \rightarrow \infty$, we obtain

$$
\eta(x)=\frac{F^{2}}{F^{2}-1} h(x) .
$$

The ratio of the maximum height between the bottom topography and the surface elevation depends on the Froude number of the incoming flow. For supercritical flow $F>1$, the surface elevation is positive with amplitude decreasing by increasing $F$. This can be compared to the solution of the steady fKdV model, having a branch of solution with different amplitude, see for example in the work of Shen et al. [4] and Wiryanto [9]. Results of (19) indicate that the fKdV solution with high amplitude is unstable. In contrary for subcritical flow $F<1$, the elevation is negative with decreasing amplitude for decreasing $F$.

\section{Numerical Procedure}

Now we describe a numerical procedure for solving (17) and (18). The procedure is similar to the one used by Wiryanto [10] to solve an interfacial wave model. We write them as

$$
\begin{gathered}
\eta_{t}=\mathcal{G}\left(\eta, \eta_{x}, u_{x}, u_{x x}, h, h_{x}\right), \\
u_{t}=\mathcal{H}\left(\eta_{x}, u, u_{x}\right),
\end{gathered}
$$

where $\mathcal{G}$ and $\mathcal{H}$ are given by

$$
\mathcal{G}=-F\left(\eta_{x}+u_{x}-h_{x}\right)-\epsilon F\left(\eta_{x} u_{x x}+u_{x} \eta-u_{x} h\right),
$$




$$
\mathcal{H}=-F u_{x}-\frac{1}{F} \eta_{x}-\epsilon F u u_{x} .
$$

The space domain is discretized uniformly into a finite number of nodes, $x_{i}=$ $i \Delta x$, where $i=0,1,2, \ldots, N-1$ for some $N$. Here $\Delta x$ is the space step. The time domain is discretized into $t^{n}=n \Delta t$, where $n=0,1,2, \ldots$ The notation $\Delta t$ represents the time step.

We propose to solve the model (17) and (18) using an explicit predictorcorrector method. The predictor step uses the third order Adams-Bashforth formulation

$$
\begin{aligned}
{ }^{*} \eta_{i}^{n+1} & =\eta_{i}^{n}+\frac{\Delta t}{12}\left[23 \mathcal{G}_{i}^{n}-16 \mathcal{G}_{i}^{n-1}+5 \mathcal{G}_{i}^{n-2}\right] \\
{ }^{*} u_{i}^{n+1} & =u_{i}^{n}+\frac{\Delta t}{12}\left[23 \mathcal{H}_{i}^{n}-16 \mathcal{H}_{i}^{n-1}+5 \mathcal{H}_{i}^{n-2}\right]
\end{aligned}
$$

The values ${ }^{*} \eta_{i}^{n+1}$ and ${ }^{*} u_{i}^{n+1}$ are the predicted values of $\eta_{i}^{n+1}$ and $u_{i}^{n+1}$, respectively. These values are then used to compute ${ }^{*} \mathcal{G}_{i}^{n+1}$ and ${ }^{*} \mathcal{H}_{i}^{n+1}$, which are the predicted values of $\mathcal{G}_{i}^{n+1}$ and $\mathcal{H}_{i}^{n+1}$ respectively. The corrector step uses the third order explicit Adams-Moulton formulation

$$
\begin{aligned}
\eta_{i}^{n+1} & =\eta_{i}^{n}+\frac{\Delta t}{12}\left[5^{*} \mathcal{G}_{i}^{n+1}+8 \mathcal{G}_{i}^{n}-\mathcal{G}_{i}^{n-1}\right], \\
u_{i}^{n+1} & =u_{i}^{n}+\frac{\Delta t}{12}\left[5^{*} \mathcal{H}_{i}^{n+1}+8 \mathcal{H}_{i}^{n}-\mathcal{H}_{i}^{n-1}\right] .
\end{aligned}
$$

We use finite difference formulas to discretize equations (22) and (23). Here we use central difference approximation for the space derivatives $\eta_{x}, u_{x}, h_{x}$ and $u_{x x}$. A special treatment is applied for $n=0$, i.e. the first iteration of the method to get $\eta_{i}^{1}$ and $u_{i}^{1}$. We define $\eta_{i}^{-2}=0$ and $u_{i}^{-2}=0$, and in addition, $\eta_{i}^{-1}=0$ and $u_{i}^{-1}=0$. Note that the initial condition is given by $\eta_{i}^{0}=0$ and $u_{i}^{0}=0$.

\section{Numerical Results}

Most calculations of the numerical procedure described above use $N=2000$ number of nodes, with $\Delta x=0.1, \Delta t=0.001$ and parameter $\epsilon=0.1$. The disturbance of the flow is a bump placed at the middle of the domain, presented as a smooth function, such as

$$
h(x)=s \operatorname{sech}^{2}\left(b\left(x-x_{o}\right)\right)-\alpha
$$

The height and width of the bump are presented by $s$ and $b$. The value $x_{o}$ is the position of the crest of the bump. The constant $\alpha$ is required to get zero disturbance at the left and right ends of the interval, $h(0)=0=$ $h(200)$. As the initial condition, the water surface and the particle velocity are 
zero, $\eta(x, 0)=0$ and $u(x, 0)=0$. These are followed by analytical boundary conditions $\eta(0, t)=0$ and $u(0, t)=0$, as the left end of the domain is assumed that the flow is uniform.

The numerical calculation is performed by plotting the surface elevation $\eta(x, t)$. For some different times, the surface elevation is shifted upward, so that we can observe the wave generation and its propagation. Typical plots of surface elevation $\eta$ for supercritical flow are shown in Figure 2, corresponding to $F=1.5$ in Figure 2(a) and $F=4.5$ in Figure 2(b). After long run, the negative-amplitude waves have run away from the fluid domain, and solitarylike elevation is as the steady solution, as shown in Figure 2(b). We calculate the plots in Figure 2 using $F=1.5$ with $s=0.1, b=0.3$ and $\alpha=0$, so the bottom topography is

$$
h(x)=0.1 \operatorname{sech}^{2}(0.3(x-100))
$$

With this topography, the values of $h(0)$ and $h(200)$ are of the order $O\left(10^{-27}\right)$, which is zero numerically. This means that the condition $h(0)=0=h(200)$ is computationally satisfied. The disturbance of the flow causes the surface is lifted up, and increasing the height above the bump, as shown in Figures 2(a) and 2(b), followed by propagating the wave, which is generated on the right side of the bump. The left and right boundaries are set to be absorbing (transmissive) boundaries by linear extrapolation of $\eta$ and $u$. Therefore, the chosen space domain $[0,200]$ is sufficient in the computation. In general, the surface elevation contains one crest and two troughs. As time increases, the surface profile tends to steady with solitary-like. This limiting surface confirms to a solution of the forced KdV model equation, obtained in the work of Shen et al. [4] as well as Wiryanto [9]. In Figure 2, we can compare the wave propagation between $F=1.5$ and $F=4.5$, that the wave speed is larger for larger Froude number. It can be seen the tangential of the wave propagation, the right going waves reach the right boundary faster for larger Froude number.

From the wave evolution in Figure 2(a), we collect the maximum value of $\eta$, namely $\eta_{\max }$ for some values $t$, and we observe that $\eta_{\max }$ increases asymptotically by increasing the time, higher than the bottom height. We show in Figure 3(a) the plot of $\eta_{\max }$ versus time $t$ asymptotically. After $t>25$, $\eta_{\max }$ relatively does not change much, i.e. $\eta_{\max } \approx 0.1833$. If we compare $\eta_{\max }$ with the bottom height, $\eta_{\max }$ is slightly higher than $F^{2} /\left(F^{2}-1\right) h_{\max }$, the maximum value of $\eta$ for linear steady solution in (19). The value $h_{\max }$ is the maximum bottom topography of $h(x)$. The surface elevation for that large $t$ is solitary-like shown in Figure 3(b). Similarly for $F=4.5$, for long run we obtain the solitary-like profile, with $\eta_{\max }$ smaller than the result from $F=1.5$, but approximating to $F^{2} /\left(F^{2}-1\right) h_{\max }$.

Now, we present the numerical solution of the model (17) and (18) for subcritical flow $F<1$. The uniform velocity is less stronger than the gravity 


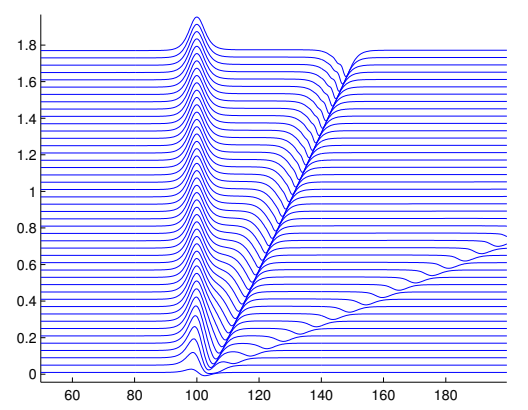

(a)

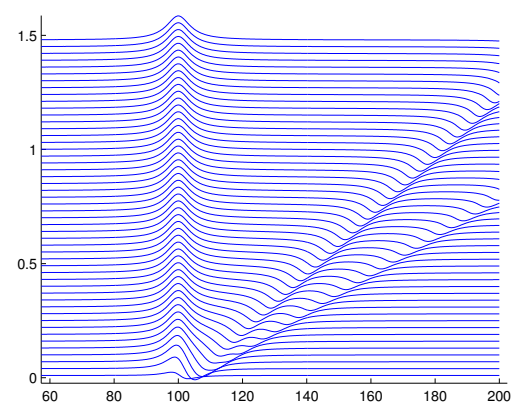

(b)

Figure 2: Plot $\eta(x, t)$ for different values $t$, (a). Calculated using $F=1.5$, (b). $F=4.5$.

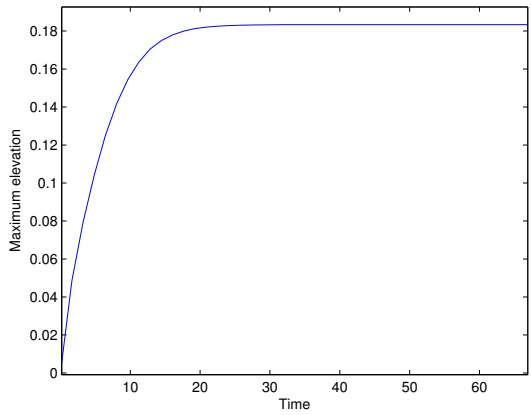

(a)

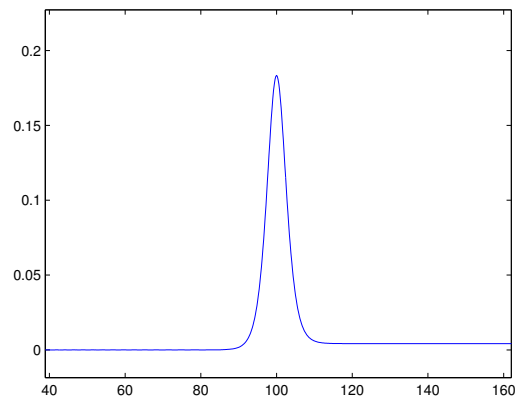

(b)

Figure 3: (a). Plot $\eta(x, 160)$, calculated using $F=1.5$, (b). Plot $\eta_{\max }$ versus time $t$.

wave speed, so that the flow is partly reflected by the disturbance, and the positive surface-wave propagates to the left. This then interacts to the incoming flow, so that the assumption uniform flow at the upstream is not longer valid. Therefore, the forced KdV model should be revised for subcritical case. In Figure 4, we present the wave generation and the propagation. We calculate the plot for $F=0.6$. The positive elevation propagates to the left, and one of the negative-amplitude waves stays above the bump. The calculation is stopped when the left propagation is close to the left boundary. For smaller Froude numbers, the wave speed increases for the positive-amplitude wave to the left. The steady profile is obtained for $t \rightarrow \infty$, similar to the bump with negative elevation. In propagation of the positive-amplitude wave to the left, a train of waves appears behind the main positive-amplitude wave. We show in Figure 4(a) by amplifying Figure 4(b) around the main wave to confirm that 
the train of waves is not an error of the calculation.

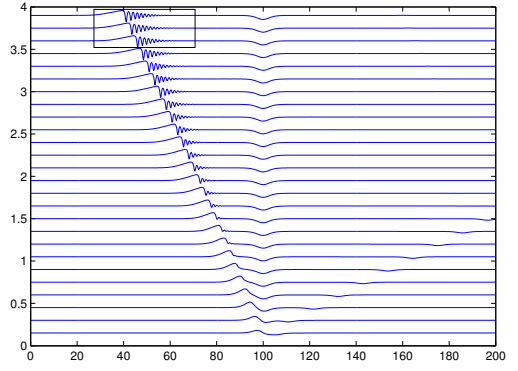

(a)

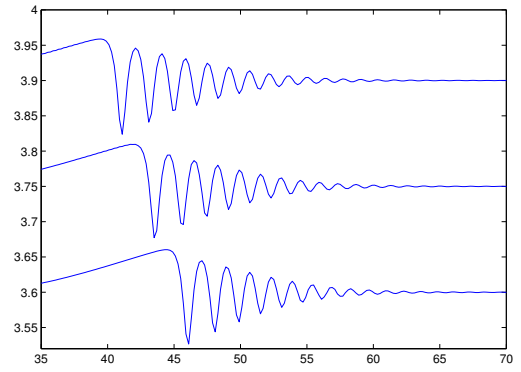

(b)

Figure 4: (a). Surface elevation $\eta(x, t)$ for $F=0.6$. (b). The zoom of the rectangular-signed inlet in (a).

Similar results, wave generation and its propagation can be observed for other bottom topography, for example a cosine bump in a certain interval and zero in outside that interval. From this unsteady model, a unique solution is obtained. This can be used to identify which stable solution is for the steady model, as there are two solutions for a given bump with supercritical Froude number; see $[1,4,9]$.

\section{Conclusions}

We have derived a Bousinesq-type model for surface wave, generated by flow disturbed by a bump. The unsteady model was solved numerically by a predictor-corrector method, to observe the wave propagation, depending to the incoming flow, presenting by Froude number. As the flow is disturbed by a bump, a wave containing a crest and two troughs is generated. For supercritical flow, both troughs propagate to the right, and the crest is left above the bump, confirms to the result in steady case. Meanwhile, for subcritical the steady case could not be obtained by an fKdV model, as the wave with the crest is going to the left and interacts to the incoming flow.

Acknowledgements. The research of the problem was supported by Decentralization Research-Grants, contract numbers 1063b/I1.C01.2/PL/2014, under Principal Investigator L.H. Wiryanto and 1063c/I1.C01.2/PL/2014, under Principal Investigator S. R. Pudjaprasetya. The grants are mainly for the first author, and they are from Directorate General of Higher Education, Ministry of Education and Culture-Indonesia. The second author thanks the 
Australian National University and SMERU Research Institute for the financial support through the Indonesia Project Research Grant 2013/2014.

\section{References}

[1] L.K. Forbes and L.W. Schwartz, Free surface flow over a semicircular obstruction, J. Fluid Mech., 114 (1982), 299-314. http://dx.doi.org/10.1017/S0022112082000160

[2] J.M. Vanden-Broeck, Free surface flow over an obstruction in a channel, Phys. Fluid, 30 (1987), 2315-2317. http://dx.doi.org/10.1063/1.866121

[3] L.K. Forbes, Critical free-surface flow over a semi-circular obstruction, $J$. Eng. Maths., 22 (1988), 3-13. http://dx.doi.org/10.1007/BF00044362

[4] S.P. Shen, M.C. Shen and S. M. Sun, A model equation for steady surface waves over a bump, J. Eng. Maths., 23 (1989), 315-323. http://dx.doi.org/10.1007/BF00128905

[5] S.S.P. Shen, Forced solitary waves and hydraulic falls in two-layer flows, J. Fluid Mech., 234 (1992), 583-612. http://dx.doi.org/10.1017/S0022112092000922

[6] F. Chardar, F. Dias, H.Y. Nguyen and J.M. Vanden-Broeck, Stability of some stationary solutions to the forced $\mathrm{KdV}$ equation with one or two bumps, J. Eng. Maths., 70 (2011), 175-189. http://dx.doi.org/10.1007/s10665-010-9424-6

[7] R. Camassa and T.Y. Wu, Stability of forced steady solitary waves, Phil. Trans. Royal Soc. A, 337 (1991), 429-466. http://dx.doi.org/10.1098/rsta.1991.0133

[8] R. Camassa and T.Y.T. Wu, Stability of some stationary solutions for the forced KdV equation, Physica D, 51 (1991), 295-307, http://dx.doi.org/10.1016/0167-2789(91)90240-A

[9] L.H. Wiryanto, Numerical solution of a KdV equation, model of a free surface flow, Applied Math. Sci., 8 (2014), 4645-4653. http://dx.doi.org/10.12988/ams.2014.46404

[10] L.H. Wiryanto, Numerical solution of Boussinesq equations as a model of interfacial-wave propagation, Bul. Malay. Math. Sci. Soc., 28 (2005), $163-172$.

Received: July 11, 2014 